\title{
Depression of IncRNA MINCR antagonizes LPS-evoked acute injury and inflammatory response via miR-146b-5p and the TRAF6-NFkB signaling
}

Wei Gao ${ }^{1}$ and Ying Zhang ${ }^{2^{*}}$ (1)

\begin{abstract}
Background: Inflammation plays an important role in the development of acute lung injury (ALI) and acute respiratory distress syndrome (ARDS). The long non-coding RNA (InCRNA) MINCR is closely related to inflammation injury. This study was performed to explore the protective effects and mechanisms of MINCR in lipopolysaccharide (LPS)induced lung injury and inflammation.
\end{abstract}

Methods: The expression levels of MINCR and miR-146b-5p in lung tissue status were detected by using quantitative real-time polymerase chain reaction (qRT-PCR), hematoxylin and eosin staining, immunohistochemical staining, and terminal deoxynucleotidyl transferase dUTP nick end labeling assay. Enzyme-linked immunosorbent assay and Western blotting analysis were used to detect the expression of inflammatory factors such as tumor necrosis factor (TNF)- $a$, interleukin (IL)-6, and IL-10 in lung tissue. The relationship between MINCR, miR-146b-5p, and TRAF6 was explored using bioinformatics analysis and luciferase assay.

Results: The expression levels of MINCR were increased in a mouse model of LPS-induced ALI and small airway epithelial cells (SAECS). shMINCR resulted in increased cell viability and decreased apoptosis, which protected against LPS-induced cell damage. shMINCR can inhibit the formation of neutrophil extracellular traps, neutrophil numbers, myeloperoxidase activity, and the production of inflammatory cytokines IL-6, IL-1 $\beta$, and TNF-a induced by LPS. The silencing of miR-146b-5p reversed the effects of MINCR on LPS-induced lung damage. Sh-MINCR decreased the expression levels of TRAF6 and p-P65 in LPS-induced SAECs and lung tissues. Co-transfection of sh-MINCR with miR146b-5p inhibitor reversed the effect of sh-MINCR on the expression of TRAF6 and p-P65.

Conclusions: MINCR may induce alveolar epithelial cell injury and inflammation and aggravate the progression of ALI/ARDS through miR-146b-5 $p$ and TRAF6/NF-KB pathways, which would provide a promising target for the treatment of ALI/ARDS.

Keywords: MINCR, miR-146b-5p, TRAF6/NF-kB, LPS, ALI/ARDS

*Correspondence: YingZhangShando@163.com

${ }^{2}$ Department of Respiratory, The Second Hospital of Shandong

University, No.247 Beiyuan Avenue, Jinan 250033, Shandong, People's Republic of China

Full list of author information is available at the end of the article

\section{Background}

Acute lung injury (ALI) is a disease that is widely distributed worldwide. It is an acute progressive insufficiency or respiratory failure caused by various internal and external factors of the lung, with a mortality rate of up to $40 \%$ (Tao et al. 2019). The common pathological manifestations of 
ALI are damage to alveolar epithelial cells, an increase in pulmonary capillary permeability, thickening of the alveolar wall, and the release of inflammatory factors in the alveoli cavity (Ochroch et al. 2019). With reduced lung volume, reduced lung compliance can develop into acute respiratory distress syndrome (ARDS) in later stages (Cao et al. 2018). The pathogenesis of ARDS is affected by coagulation and fibrinolysis. The function of the human coagulation system is enhanced, whereas the fibrinolysis system is inhibited, leading to a large area of thrombosis and a large amount of fibrin deposition, resulting in vascular blockage and microcirculation structure damage (Bender et al. 2020). The lipopolysaccharide (LPS)induced lung injury model has been widely used in studies on lung injury (Zhu et al. 2013). There are no specific response methods for ALI. Currently, auxiliary ventilation and drug therapy are the main clinical response methods (Chiumello et al. 2019). Therefore, it is important to elucidate the regulatory mechanisms and signaling pathways involved in the pathological process of ALI to provide targeted drugs for clinical treatment.

At present, the pathogenesis of ALI is considered to be an inflammatory response of the body. Infection is a common risk factor for ALI, and LPS is the main endotoxin (Cheung et al. 2018). Long non-coding RNAs (lncRNAs) play key roles in regulating inflammatory immune genes (Wu et al. 2018). These can act as regulatory factors and exert their functions by interacting with proteins, RNAs, and DNA to regulate their expression at transcriptional and post-transcriptional levels (Liu et al. 2009). Several studies have investigated the regulation of lncRNAs on epigenetics and gene transcription and their involvement in disease occurrence and development, particularly in Alzheimer's disease, cancer, and other diseases (Wang et al. 2017; Xie et al. 2019). lncRNAs also play roles in controlling the balance of inflammatory responses (Fava et al. 2017). For example, lncRNA CASC2 reduces apoptosis of pulmonary epithelial cells and improves ALI by regulating the miR-144-3p/AQP1 axis ( $\mathrm{Li}$ et al. 2018a). A better understanding of lncRNAs will provide new ideas for exploring the nature of sepsis and regulating the uncontrolled inflammatory cascade in sepsis. The IncRNA MINCR is a newly identified lncRNA that is abnormally expressed in many diseases (Hu et al. 2018). For example, the expression levels of MINCR are significantly increased in primary liver cancer tissues (Lyu et al. 2019). However, the functions and mechanisms of MINCR in ALI have not yet been studied.

MicroRNAs (miRNAs) are involved in many important pathophysiological processes. In recent years, studies have explored their regulation in tumors, inflammation, and other aspects in molecular biology, cell biology, and clinical medicine (Emma et al. 2020). miRNAs are widely involved in the immune regulation of systemic inflammatory response syndrome and ALI (Iacona et al. 2018). In the process of the systemic inflammatory response, miRNAs regulate the expression of various inflammatory genes and transcription factors, the proliferation of various inflammatory cells, the production and release of various cytokines and inflammatory mediators, and the expression of various inflammatory factor receptors (Sedgeman and Michell 2019). Studies have found that miR-155, miR-17-92, and miR-223 participate in the regulation of ALI caused by toxicosis (Feng et al. 2017). MiR-146b-5p plays different roles in the progression of different diseases (Sheng et al. 2018). For example, it can inhibit the proliferation of osteosarcoma cells (Zhu et al. 1136). However, the expression and function of miR-146b-5p in ALI remain unclear. NF- $\mathrm{kB}$ is a crucial factor in cell proliferation and differentiation (Jian et al. 2019). It acts on the rank receptor on the cell membrane and transmits the signal to TRAF6, which is an important link molecule in cell formation and can cause the chain reaction of downstream signaling and promote its proliferation (Kanaya et al. 2018). Isoprolactone has been reported to inhibit TRAF6 ubiquitination and reduce ALI development (Ding et al. 2019). Therefore, it was speculated that MINCR regulated the TRAF6-NF-kB axis through miR-146b-5p to regulate LPS-induced ALI. This study explored the mechanism of action of MINCR in ALI.

\section{Materials and methods}

\section{Ethics statement}

All animal experiments were performed in accordance with the Guidelines for the Care and Use of Laboratory Animals (revised in 1996, publication No. 85-23) published by the National Institutes of Health, Nursing committee approval.

\section{Animal model of sepsis-induced ALI}

Male C57BL/6 mice ( $\mathrm{N}=10,6-8$ weeks old, 20-24 g) were obtained from Wild River Laboratory Animal Technology Co., Ltd. (Beijing, China). The mice were randomly fed food and water. They were treated with $5 \mathrm{mg} /$ $\mathrm{kg}$ LPS (Escherichia coli O111:B4 dissolved in saline, Sigma, St. Louis, MO, USA) by intratracheal instillation to induce ALI. The control group mice were anesthetized and sacrificed 24, 48, and $72 \mathrm{~h}$ after LPS administration. The mice were intraperitoneally injected with $30 \mathrm{mg} /$ kg pentobarbital (WS20060401, Sinopharm Chemical, China). Bronchoalveolar lavage fluid (BALF), plasma, and tissue samples were collected by lavaging the lung with $1 \mathrm{~mL}$ PBS two times, and the red blood cells were lysed using lysis buffer followed by centrifugation. The trachea was intubated and the thorax was opened through 
a midline incision. The BALF was centrifuged, and the supernatant without cells was stored at $-80{ }^{\circ} \mathrm{C}$ for enzyme-linked immunosorbent assay (ELISA) as previously described (Gregoire et al. 2017, 2018).

\section{Lung histology and lung injury score}

Lung tissues were collected from the mice $48 \mathrm{~h}$ after LPS instillation. The lungs were inflated with $10 \%$ buffered formalin, fixed with $10 \%$ buffered formalin, embedded in paraffin, and cut into 5- $\mu \mathrm{m}$ sections. The tissue sections were stained with hematoxylin and eosin (H\&E), evaluated, and scored by a pathologist blinded to the experimental groups. To evaluate lung injury, seven independent random lung fields were evaluated per mouse for neutrophils in alveolar spaces, neutrophils in the interstitial spaces, hyaline membranes, proteinaceous debris filling the airspaces, and alveolar septal thickening, and weighted according to the relevance ascribed by the official American Thoracic Society workshop report on features and measurements of experimental acute lung injury in animals (Sheng et al. 2018). The resulting injury score was a continuous value between 0 and 1 .

\section{Histological evaluation of lung injury}

The lower right lung lobe was cut into thin sections $(5 \mathrm{~mm})$. The number of alveolar sacs was determined after $H \& E$ staining by investigators blinded to the experimental groups. Three lung sections from each mouse were examined. Three high-power fields (X100) were randomly selected in each section for observation. The average number of alveolar sacs per mouse was determined as the sum of all fields divided by 9 . The crowded area was defined as thickened septa in the lung parenchyma where alveolar sacs were partly or completely collapsed under H\&E-stained sections. A scoring system was used to quantify the crowded area by investigators blinded to the experimental groups, defined as follows: 0 , no crowded area; $1,15 \%$ of the area crowded.

High-power field was a crowded area; $2,15-25 \%$ of the area crowded; $3,25-50 \%$ of the area crowded; $4,50-75 \%$ of the area crowded; 5, 75-100\% of the area crowded.

Caspase-3 (Beyotime, Shanghai, China) activities were measured using colorimetric assay kits following the manufacturer's instructions.

\section{Adenovirus gene delivery}

Recombinant adenovirus containing mouse MINCR shRNA (Ad-MINCR-shRNA) and Ad-GFP were obtained from Obio Company (Shanghai Obio Technology Co., Ltd.). Seven days before ALI induction, adenovirus $\left(1 \times 10^{9}\right.$ transducing units [TU], $\left.50 \mu \mathrm{g} / 0.2 \mathrm{~mL}\right)$ was intravenously injected into mice. Control adenovirus (AdGFP) was injected into the control group. MiR-146b-5p antagomir, miR-146b-5p mimic, and NC were purchased from GenePharma (Shanghai, China). Thereafter, $50 \mu \mathrm{g}$ of miR-146b-5p antagomir, miR-146b-5p mimic, or its negative control, was dissolved in $200 \mu \mathrm{L}$ of sterile doubledistilled water, and $50 \mu \mathrm{L}$ of glucose solution was added to it. The tail vein of the mice was injected with $200 \mu \mathrm{L}$ working solution (Chen et al. 2018; D’Alessio et al. 2016).

\section{Cell culture and LPS exposure}

The human lung epithelial cell line small airway epithelial cells (SAECs) were obtained from Procell (Wuhan, China). SAECs were cultured in human collagen type IVcoated flasks (Sigma, St. Louis, MO, USA) supplemented with SAGM Bullet kit culture medium (Lonza, Allendale, NJ, USA). The cells were stored in DMEM supplemented with $10 \%$ fetal bovine serum (FBS). Adenovirus $\left(1 \times 10^{9}\right.$ $\mathrm{TU}, 100 \mathrm{nmol} / \mathrm{L}$ ) was used to infect the cells. The cells were cultured in media containing different doses of LPS.

\section{Cell viability assay}

Cells were seeded into a 96-well plate at a density of 5000 cells/well. Thereafter, $100 \mu \mathrm{L}$ of CCK8 solution (Liji, Shanghai, China) was added. After $4 \mathrm{~h}$, the absorbance at $450 \mathrm{~nm}$ was measured using a microplate reader (Bio Tek Instruments, USA).

\section{Apoptosis assay}

Cells were plated into 6-well plates at a density of $5 \times 10^{5}$ cells/well and counted. The cells were centrifuged and $195 \mu \mathrm{L}$ of Annexin V-FITC binding solution was added to them. After mixing with $5 \mu \mathrm{L}$ of Annexin V-FITC and $10 \mu \mathrm{L}$ propidium iodide staining solution, the cells were incubated for 10-20 min.

\section{Myeloperoxidase (MPO) activity analysis}

The lung tissues preserved at $-80^{\circ} \mathrm{C}$ were removed, liquid nitrogen was added to them, and they were rapidly grounded to powder in a mortar. Thereafter, $300 \mu \mathrm{L}$ of RIPA lysate containing PMSF inhibitor was added. The sample was then placed on an ice plate for cracking for $10 \mathrm{~min}$, and the lysate was collected after the sample was fully cracked. The collected lysate was centrifuged at $4{ }^{\circ} \mathrm{C}$ at $12,000 \mathrm{rpm}$, and the collected supernatant was carefully sucked. The concentrations of all samples were adjusted using RIPA lysate and detected using an MPO detection kit.

\section{ELISA}

The expression levels of interleukin (IL)-6, tumor necrosis factor (TNF)- $\alpha$, and IL-10 were determined using a kit (Fisher Scientific, Waltham, MA, USA). A Luminex 


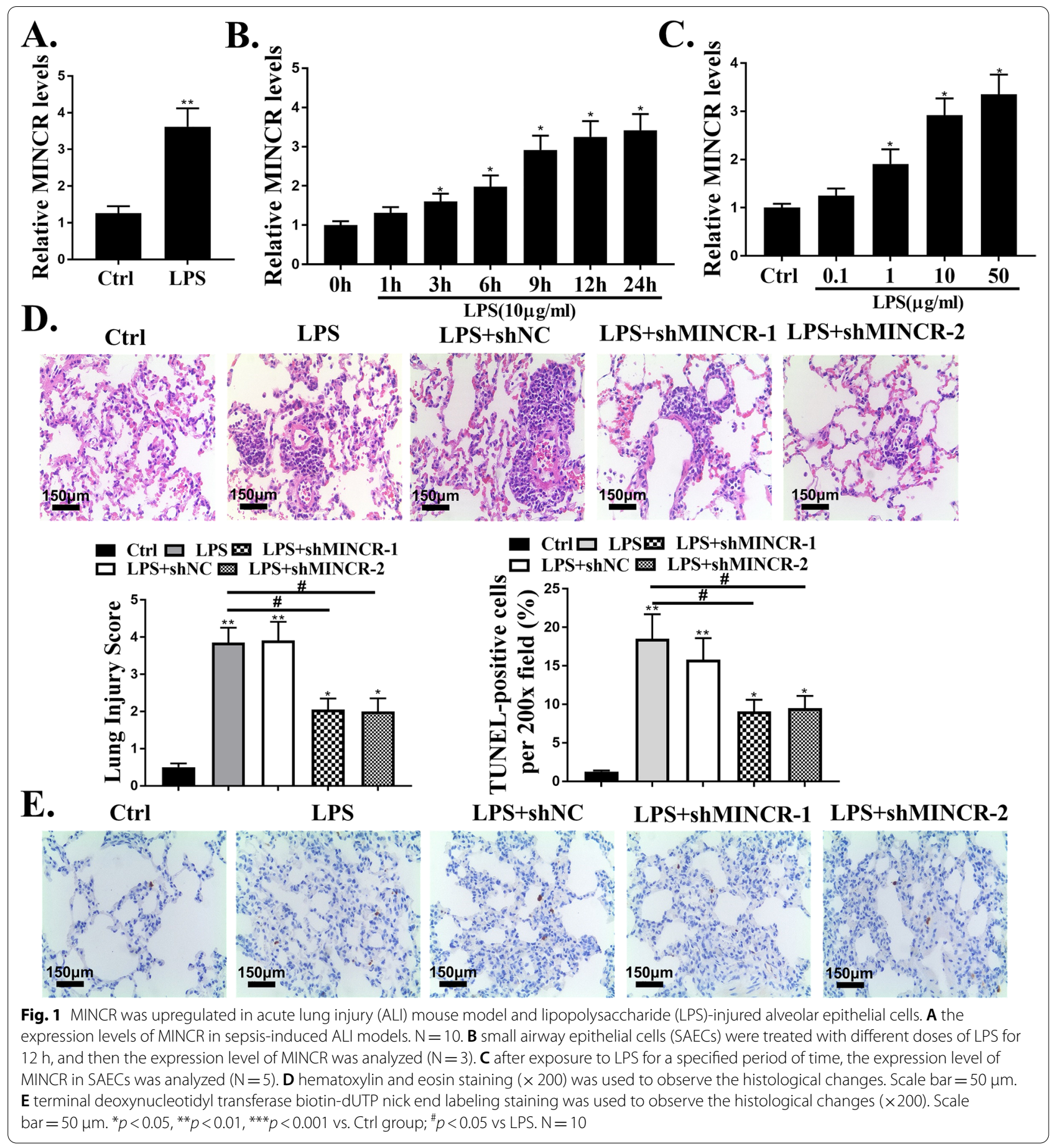

100/200 photometer (Luminex, Austin, Texas, USA) was used for cytokine quantification.

Polymorphonuclear leukocyte (PMN) isolation and culture BALF neutrophils were isolated by centrifugation. PMNs were collected and purified. Neutrophils were cultured in RPMI 1640 medium (Weike, Shanghai, China) containing 10\% FBS.

\section{Western blotting analysis}

Transfected cells were collected, and total proteins were extracted. Protein concentrations were determined 


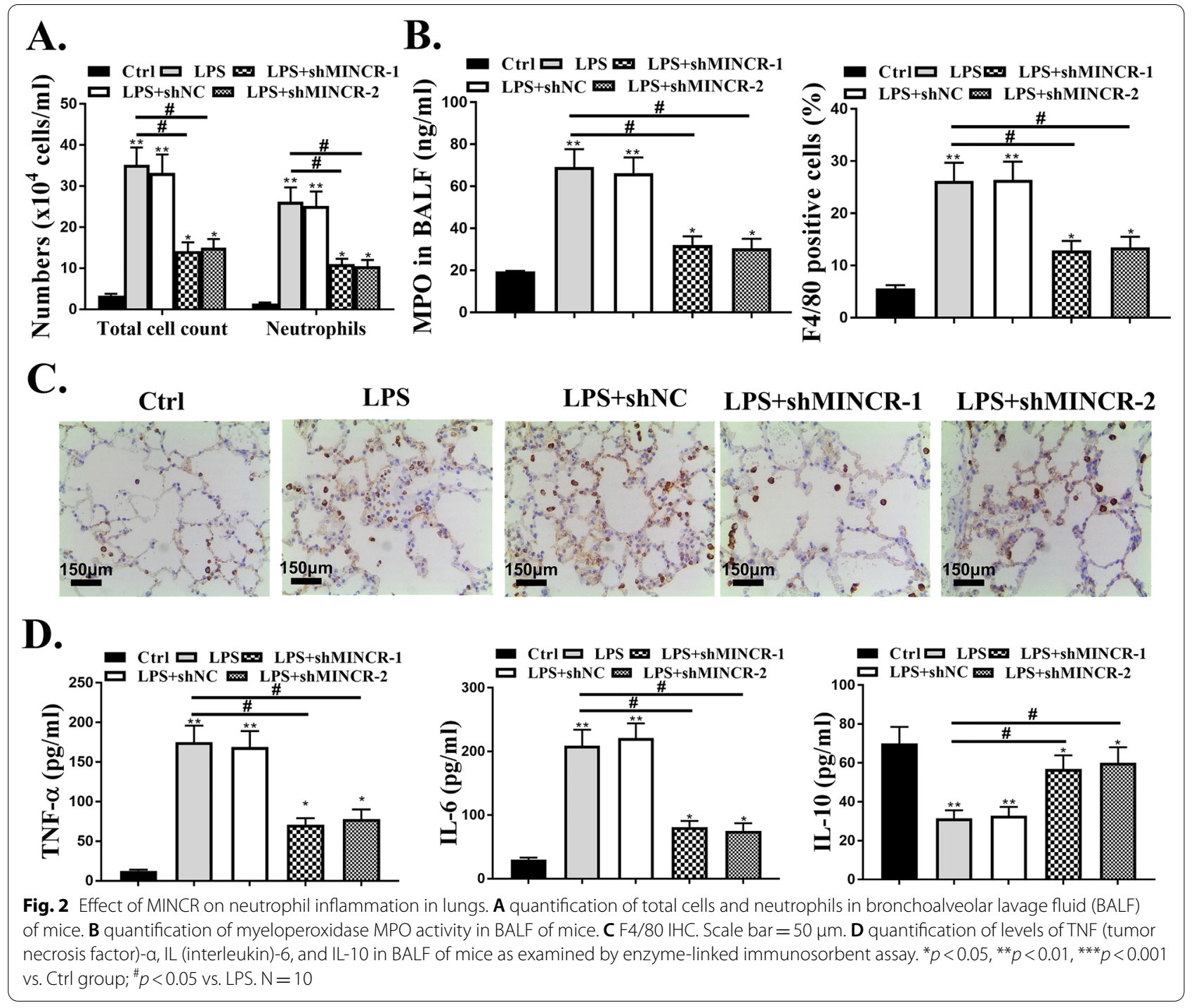

using a bicinchoninic acid protein assay kit. Thereafter, proteins were separated using $10 \%$ polyacrylamide gel electrophoresis and blocked with 5\% BSA for $1 \mathrm{~h}$. After transferring onto PVDF membrane, the membrane was incubated with anti-TRAF6 (1:1000, Amyjet, Wuhan, China), anti-p65 (1:1000, Amyjet, Wuhan, China), anti-p-p65 (1:1000, Amyjet, Wuhan, China), anti-IL-10 (1:1000, Amyjet, Wuhan, China), anti-IL-6 (1:1000, Amyjet, Wuhan, China), anti-TNF- $\alpha$ (1:1000, Amyjet, Wuhan, China), and anti-GAPDH (1:1000, Amyjet, Wuhan, China) overnight, followed by incubation with anti-rabbit secondary antibody (1:1000, Amyjet, Wuhan, China) for $1 \mathrm{~h}$ (Sanaa and Seada 2020).

\section{Dual luciferase report assay}

The wild type (WT) 3'-UTR of MINCR cDNA was synthesized by polymerase chain reaction (PCR) and cloned into pMIR-REPORT luciferase to generate the WT-MINCR 3'-UTR. MINCR 3'- was generated based on the WT-MINCR $3^{\prime}$-UTR A mutant of UTR and named as the resulting vector MUT-MINCR $3^{\prime}$-UTR. These vectors (pMIR-REPORT plasmid, WT-MINCR $3^{\prime}$-UTR, or MUT-MINCR $3^{\prime}$-UTR) and miR-146b-5p Mock or miR NC were transiently transfected into cells using Lipofectamine 3000 reagent (Invitrogen, Carlsbad, CA, USA). After $48 \mathrm{~h}$ of transfection, luciferase activity was measured using Promega (Madison, WI, USA). The putative binding site of TRAF6 to miR146b-5p was also detected using the same method.

\section{RNA pull-down assay}

Cells were transfected with biotin-labeled Bio-miR146b-5p or Bio-miR-146b-5p-probes and Bio-NCprobes for $48 \mathrm{~h}$ and then collected. Cells were lysed 


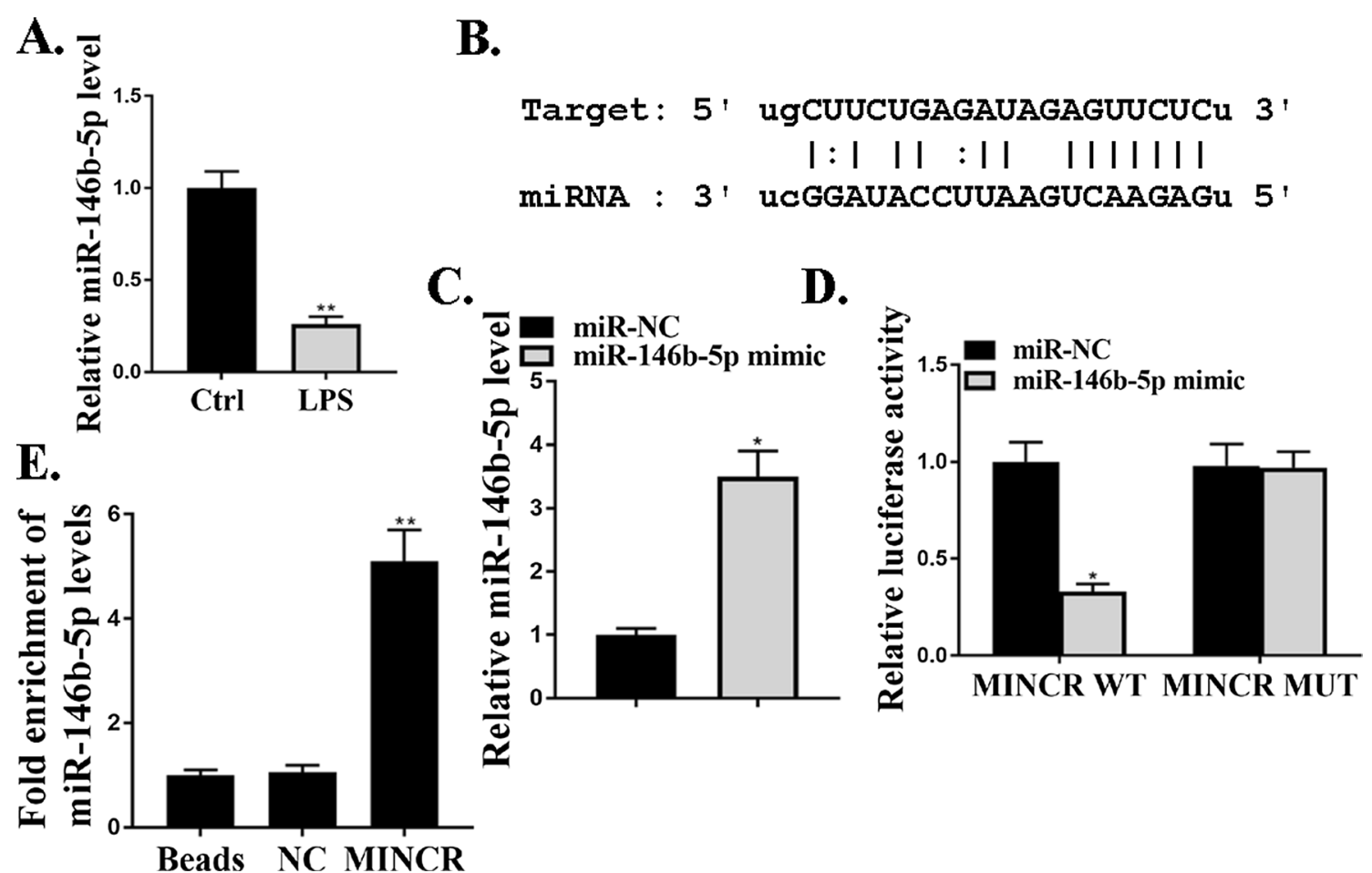

Fig. 3 MiR-146b-5p directly regulated MINCR. A miR-146b-5p expression level in a model of acute lung injury (ALI) induced by lipopolysaccharide (LPS), $N=10$. B MiR-146b-5p was predicted as a potential target of MINCR (C) miR-146b-5p expression level after mimic treatment. D analysis of luciferase activity in cells co-transfected with MINCR-wild type (WT) or MINCR-Mut vector. $\mathbf{E}$ immunoprecipitation test was used to determine the enrichment of MINCR and miR-146b-5p in the immunoprecipitation complex. Bars indicated standard deviation. ${ }^{*} P<0.05,{ }^{* *} p<0.01, N=3$

using a complete RIP lysis buffer. Cell extracts were incubated with magnetic beads conjugated with anti Ago2 or anti-IgG antibodies. RNA was then purified by qRT (quantitative reverse transcription)-PCR analysis.

\section{qRT-PCR analysis}

Total RNA was extracted using TRIzol reagent (Biosntech, Beijing, China). RNA quality was analyzed using a NanoDrop 1000 (Thermo Fisher Scientific, Inc.). SYBR Green (Takara Biotechnology, Co., Ltd., Dalian, China) was used for qRT-PCR. Amplification was performed using an ABI 7500 real-time PCR system. A qScript miRNA cDNA synthesis kit (Quantabio, Beverly, CA, USA) was used for cDNA synthesis. Gene expression levels were analyzed using the $2^{-\triangle} \triangle \mathrm{CT}$ method. The internal controls for the expression levels of miRNAs and lncRNAs were U6 and GAPDH, respectively. The primer sequences used were as follows.

\section{LncRNA MINCR, 5'-CTAATTCACCTGGCCCGA G-3' (forward)}

5'-CGGCTAGAATCCCAAGG-3' (reverse);
miR-146b-5p， 5'-CTGAATGTGAAGAGGATGT-3' (forward)

$5^{\prime}$ - G T T C T T CG G C C T C CG G GC C C- $3^{\prime}$ (reverse);

miR negative control (miR-NC), 5'-UUCUCCGAA CGUGUCACGUTT-3' (forward)

5'-UGAGAACUGAAUUCCAUAGGC UG- ${ }^{\prime}$ (reverse);

IL-1 $\beta, \quad 5^{\prime}$-CTTCAGGCAGCCAGTATCAATC-3' (forward)

5'-TGC AGT G GT CT TATG GGA ACGT-3' (reverse);

MCP1, 5'-ACAACCAAGGCCTTCCTTAC-3' (forward)

$5^{\prime}$ - TC T CAT TCC CACGAT TCC CCAG- $3^{\prime}$ (reverse);

TNF- $\alpha, \quad 5^{\prime}$-CGAGTGGCAAGCCTGGAGGCC- $3^{\prime}$ (forward)

5'-GTC TTG GA G ATC CAT CCG GT TG-3' (reverse);

GAPDH, 5'-AACGAACCTTTCATTGAC-3' (forward)

5'-CACCACTCTTACAGCAACT-3' (reverse). 


\section{Immunohistochemical staining}

The Power Vision two-step method was used and the images were analyzed using Image-Pro Plus 6.0 image color analysis software. Each slice was randomly selected from five fields of view $(\times 200$ or $\times 400)$, and the expression of F4/80 and the presence of neutrophils and macrophages were evaluated by immunohistochemistry.

\section{Terminal deoxynucleotidyl transferase biotin-dUTP nick end labeling (TUNEL) assay}

Cells from different treatment groups were collected, smeared, washed, and fixed with PBS. After reaction on ice with $100 \mu \mathrm{L}$ of permeabilization buffer for 2-5 min, the cells were washed with PBS. Thereafter, $50 \mu \mathrm{L}$ of the pre-cooled reaction solution was added to the slide and incubated for 60-90 min. Cell staining was observed using a fluorescence microscope. Positive staining with nuclear DNA fragmentation was detected by fluorescence microscopy and quantified by the number of TUNEL-positive cells in ten fields per section and at least five sections per lung (at $\times 200$ magnification).

\section{Statistical methods}

Data are represented as the mean \pm standard deviation and analyzed using SPSS19.0. Differences among multiple groups were analyzed using one-way ANOVA. The least significant difference test was used for subsequent analyses. $P<0.05$ indicating that the difference was significant.

\section{Results}

MINCR was upregulated in ALI mouse model and LPS-injured alveolar epithelial cells

Compared to the control group, the expression levels of MINCR in the lung tissue of LPS-injured mice were significantly increased $(P<0.05$, Fig. $1 \mathrm{~A})$. SAECs were exposed to LPS to simulate ALI in vitro, and the expression levels of MINCR gradually increased with an increase in the duration of LPS and an increase in the concentration of the effect $(P<0.05$, Fig. $1 \mathrm{~B}$ and $\mathrm{C})$. $H \& E$ staining results showed that the lung tissues with LPS injury showed thickened alveolar walls, pulmonary edema, and alveolar hemorrhage compared to the control group. ShMINCR significantly decreased LPS-induced lung tissue damage (Fig. 1C). Compared with the control group, the number of apoptotic cells in the lung tissues of LPS-injured mice was significantly increased $(P<0.05)$, indicating that the nuclear volume was reduced, the nuclear chromatin was concentrated and condensed, and apoptotic bodies were observed. ShMINCR significantly reduced the number of apoptotic cells in the lung tissues of mice $(P<0.05$, Fig. 1D).

\section{Downregulated MINCR reduces neutrophilic inflammation in lungs of ARDS mouse model}

Thereafter, the effect of MINCR on pulmonary neutrophil inflammation was analyzed. Compared with the control group, the total cell number and the number of neutrophils in the BALF of the LPS group were significantly increased $(P<0.05)$. Post sh-MINCR treatment, the total number of cells and the number of neutrophils in the BALF were significantly decreased $(P<0.05$, Fig. 2A). In addition, MPO activity, the number of F4/80 positive cells, and the expression levels of inflammatory factors (TNF- $\alpha$ and IL-6) in the LPS group were significantly increased $(P<0.05)$, while the expression levels of IL-10 were significantly reduced $(P<0.05)$. Post shMINCR treatment, the number of F4/80 positive cells and the expression levels of inflammatory factors (TNF- $\alpha$ and IL-6) were significantly reduced $(P<0.05)$. In contrast, the expression levels of IL-10 were significantly increased $(P<0.05$, Fig. 2B-D).

\section{MiR-146b-5p directly regulated MINCR}

The mechanism by which MINCR regulated the progression of ALI was explored. Compared to the control group, the expression levels of miR-146b-5p were significantly reduced in the lung tissues of LPS-injured mice $(P<0.05$, Fig. 3A). The online prediction tool Starbase v2.0 and miR-146b-5p was identified as a potential target of MINCR (Fig. 3B). As shown in Fig. 3C, compared to the miR-NC group, the expression levels of miR-146b-5p in the miR-146b-5p mimic group were significantly increased, indicating that the transfection was successful $(P<0.05)$. Luciferase activity was significantly reduced in cells co-transfected with miR-146b-5p and MINCR-WT $(P<0.05)$; however, the luciferase activity of MINCRMUT did not change (Fig. 3D). In addition, MINCR and miR-146b-5p were preferentially enriched in miRNAPs

\footnotetext{
(See figure on next page.)

Fig. 4 MiR-146b-5p-mediated sh-MINCR protected against lipopolysaccharide (LPS)-induced acute lung injury (ALI). A total and neutrophil counts in bronchoalveolar lavage fluid (BALF) mice. B mouse BALF myeloperoxidase (MPO) activity. $\mathbf{C}$ hematoxylin and eosin staining. Scale bar $=50 \mu \mathrm{m}$. D terminal deoxynucleotidyl transferase biotin-dUTP nick end labeling staining was used to detect apoptosis. Scale bar $=50 \mu \mathrm{m}$. E F4/80 IHC staining. Scale bar $=50 \mu \mathrm{m}$. F quantification of levels of TNF (tumor necrosis factor)-a, IL (interleukin)-6, and IL-10 were examined by enzyme-linked immunosorbent assay. ${ }^{*} p<0.05,{ }^{* *} p<0.01,{ }^{* * *} p<0.001$ vs. Ctrl group; ${ }^{*} p<0.05$ vs. LPS. $N=10$.
} 


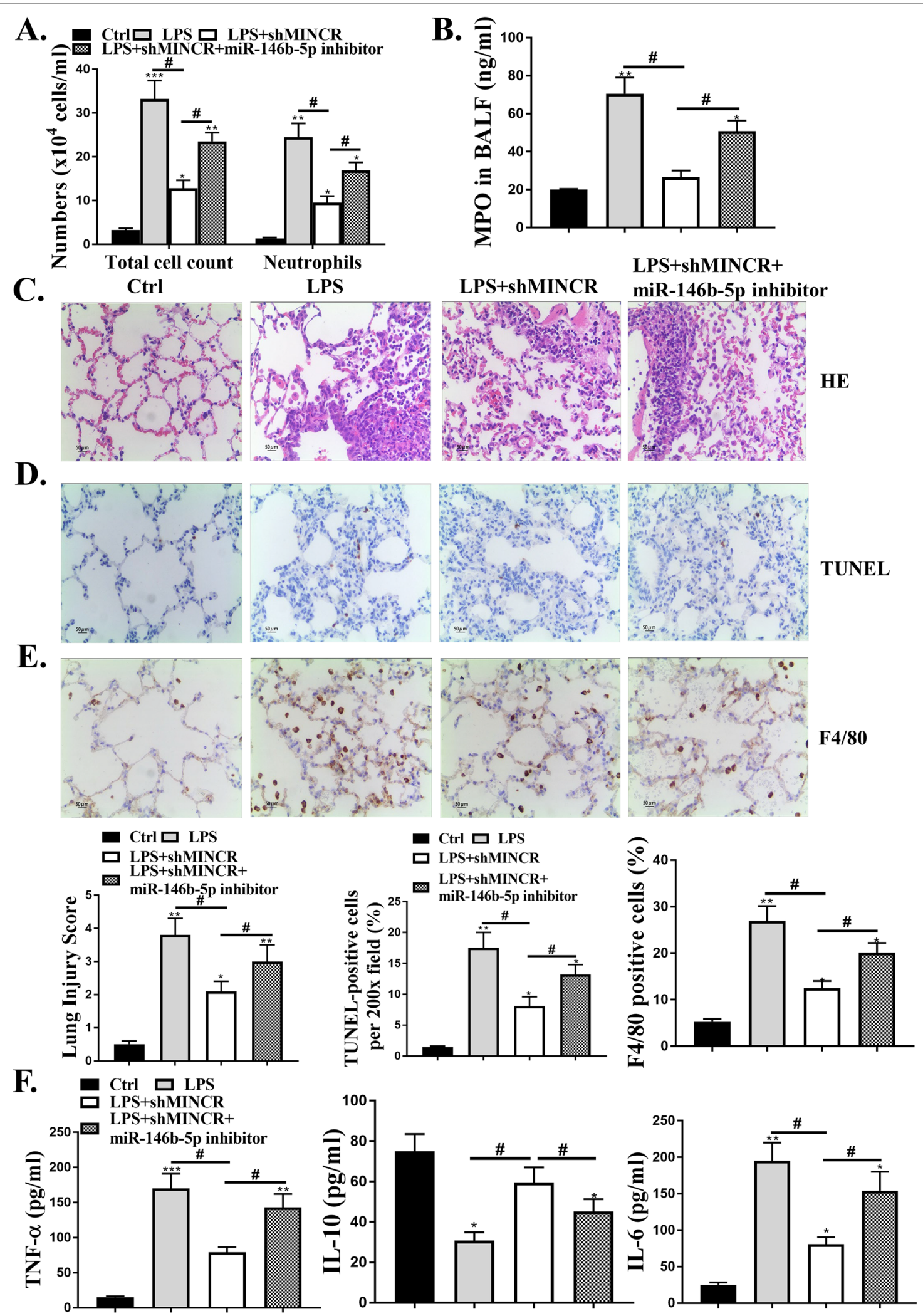

Fig. 4 (See legend on previous page.) 
containing Ago2 compared to anti-IgG immunoprecipitates (Fig. 3E). These results indicate that MINCR directly targets miR-146b-5p in ALI.

\section{MiR-146b-5p mediated the protective effects of sh-MINCR in LPS-induced ALI model}

Furthermore, we explored whether MINCR affects lung neutrophil inflammation through miR-146b-5p. shMINCR significantly reduced the total amount of BALF and neutrophil count, and MPO activity was significantly reduced after LPS treatment $(P<0.05)$. Co-transfection of Sh-MINCR with miR-146b-5p inhibitor reversed the effects of sh-MINCR on total cell number, neutrophil count, and MPO activity $(P<0.05$, Fig. $4 \mathrm{~A}$ and $\mathrm{B})$. H\&E staining results showed that shMINCR significantly reduced LPS-induced lung tissue damage, whereas cotransfection of Sh-MINCR with miR-146b-5p inhibitor enhanced lung tissue damage (Fig. 4C). shMINCR significantly reduced the number of apoptotic cells, whereas co-transfection of sh-MINCR with miR-146b-5p inhibitor increased the number of apoptotic cells in lung tissues $(P<0.05$, Fig. 4D). As shown in Fig. 4E and F, sh-MINCR significantly reduced the number of $F 4 / 80$ positive cells and the expression levels of inflammatory factors (TNF- $\alpha$ and IL-6) in vivo after LPS treatment $(P<0.05)$; the expression levels of IL-10 were significantly increased $(P<0.05)$, whereas co-transfection of sh-MINCR with miR-146b-5p inhibitor reversed the effects of sh-MINCR on MPO activity, number of F4/80 positive cells, and expression levels of inflammatory factors $(P<0.05)$.

\section{MiR-146b-5p mediated the protective effects of sh-MINCR} on LPS-induced cell apoptosis and inflammation in SAECs Compared with the control group, the SAECs in the LPS group had significantly reduced viability and increased death rate. The viability of SAECs was significantly increased and the apoptosis rate was significantly reduced after treatment with sh-MINCR, whereas cotransfection of sh-MINCR with miR-146b-5p inhibitor reversed the effect of sh-MINCR on the viability and apoptosis of SAECs $(P<0.05$, Fig. 5A and B). Caspase-3 activity was significantly elevated in the LPS group compared to the control group (Fig. 5C). In addition, compared with the control group, the expression levels of TNF- $\alpha$ and IL- 6 protein in the LPS group were significantly increased and the expression levels of IL-10 protein were significantly reduced. After treatment with sh-MINCR, the expression levels of TNF- $\alpha$ and IL- 6 proteins were significantly reduced and the expression levels of IL-10 protein were significantly increased, while cotransfection of sh-MINCR with miR-146b-5p inhibitor reversed the effect of shMINCR on the expression of cellular inflammatory factors $(P<0.05$, Fig. 5D).

\section{MiR-146b-5p directly targeted the 3'-UTR of TRAF6 mRNA}

The online prediction tool, Starbase v2.0, was used and TRAF6 was identified as a potential target for miR146b-5p (Fig. 6A). The luciferase activity of miR-146b-5p mimic and TRAF6-WT co-transfected cells was significantly reduced $(P<0.05)$; however, the luciferase activity of TRAF6-MUT did not change (Fig. 6B). Compared to the miR-NC group, the expression levels of TRAF6 protein in the miR-146b-5p group were significantly reduced $(P<0.05$, Fig. 6C). As shown in Fig. 6D and E, compared to the control group, LPS significantly increased the expression levels of TRAF6 and p-P65 in the lung tissues of SAECs $(P<0.05)$, and sh-MINCR significantly reduced the expression levels of TRAF6 and p-P65 in the lung tissues of LPS-induced SAECs, whereas co-transfection of sh-MINCR with miR-146b-5p inhibitor reversed the effect of sh-MINCR on the expression levels of TRAF6 and p-P65. These results indicated that MINCR increased the expression levels of TRAF6 and p-P65 by regulating miR-146b-5p.

\section{Discussion}

ALI/ARDS is an acute and critical illness of the respiratory system (Fu and Thangavel 2019). The imbalance of inflammatory response, increase in alveolar permeability, and abnormal activation of the coagulation pathway caused by the destruction of epithelial cells and vascular endothelial cells are the main characteristics of ALI/ ARDS pathogenesis (Tong 2018). The causes of ALI/ ARDS are diverse and their pathogenesis is highly complicated. Therefore, an in-depth understanding of the occurrence, development, and regulatory mechanisms of ALI is essential. Due to the limitation of studying the pathological changes in the lungs in vivo, animal models are the best choice to study ALI (Wang et al. 2018). Therefore, LPS was used to induce ALI in this study.

(See figure on next page.)

Fig. 5 MiR-146b-5p mediated the protective effect of sh-MINCR on lipopolysaccharide (LPS)-induced apoptosis and inflammation of small airway epithelial cells (SAECS). A cell viability was determined by CCK8. B apoptosis was detected by flow cytometry. C Caspase-3 activity assessment of different groups. D TNF (tumor necrosis factor)-a, IL (interleukin)-6 and IL-10 protein expression levels in SAECs was detected by Western blotting assay. ${ }^{*} P<0.05,{ }^{* *} p<0.01$ vs. Ctrl group; ${ }^{*} p<0.05$ vs. LPS group. $N=5$ 

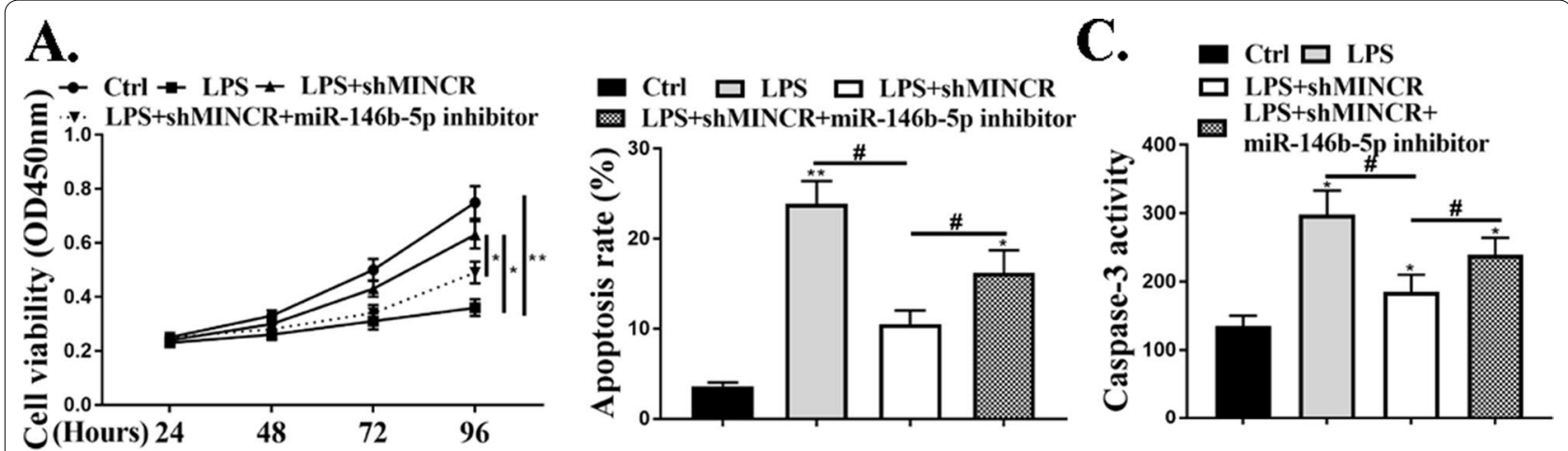

B.

Ctrl

LPS

LPS+shMINCR
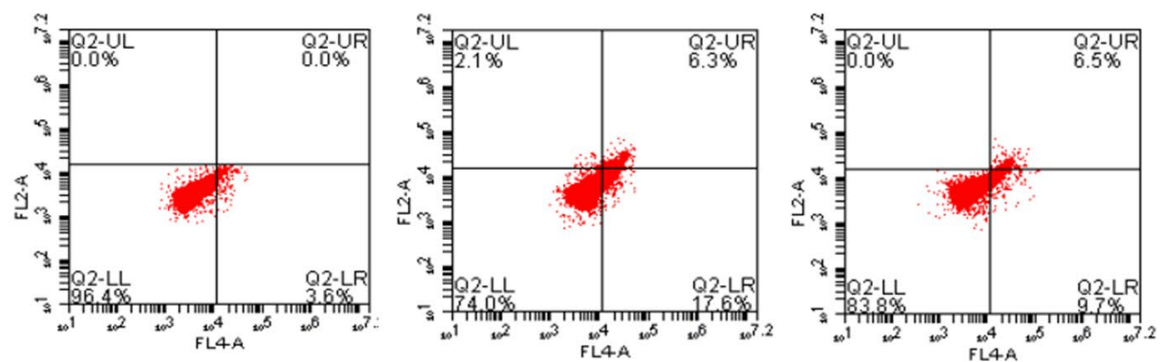

LPS+shMINCR+ miR-146b-5p inhibitor

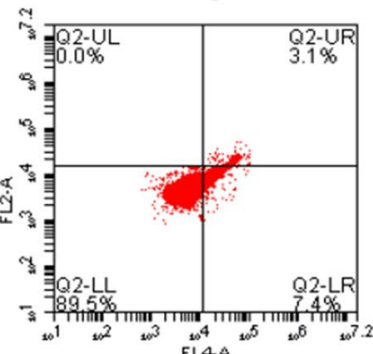

D.
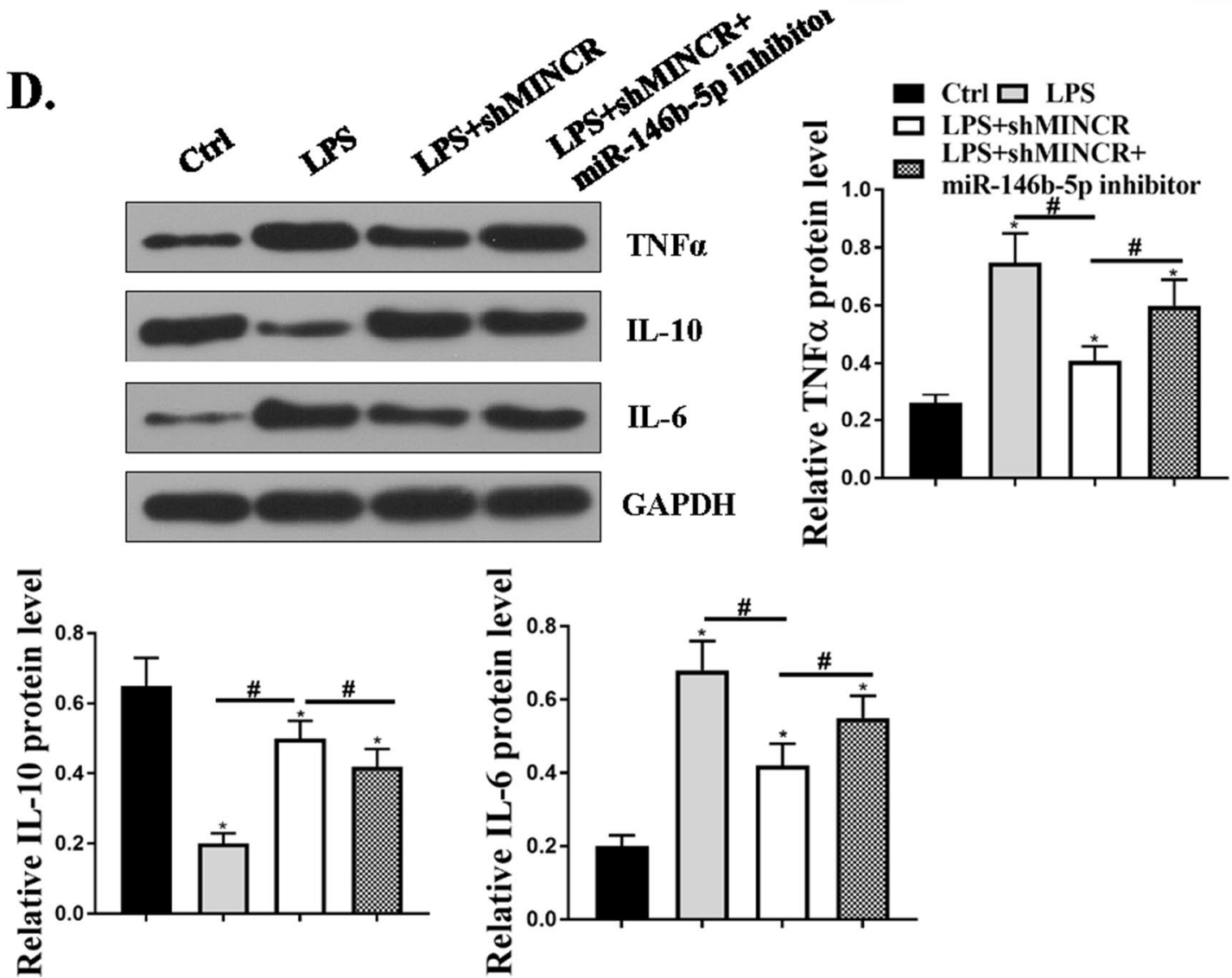

Fig. 5 (See legend on previous page.) 


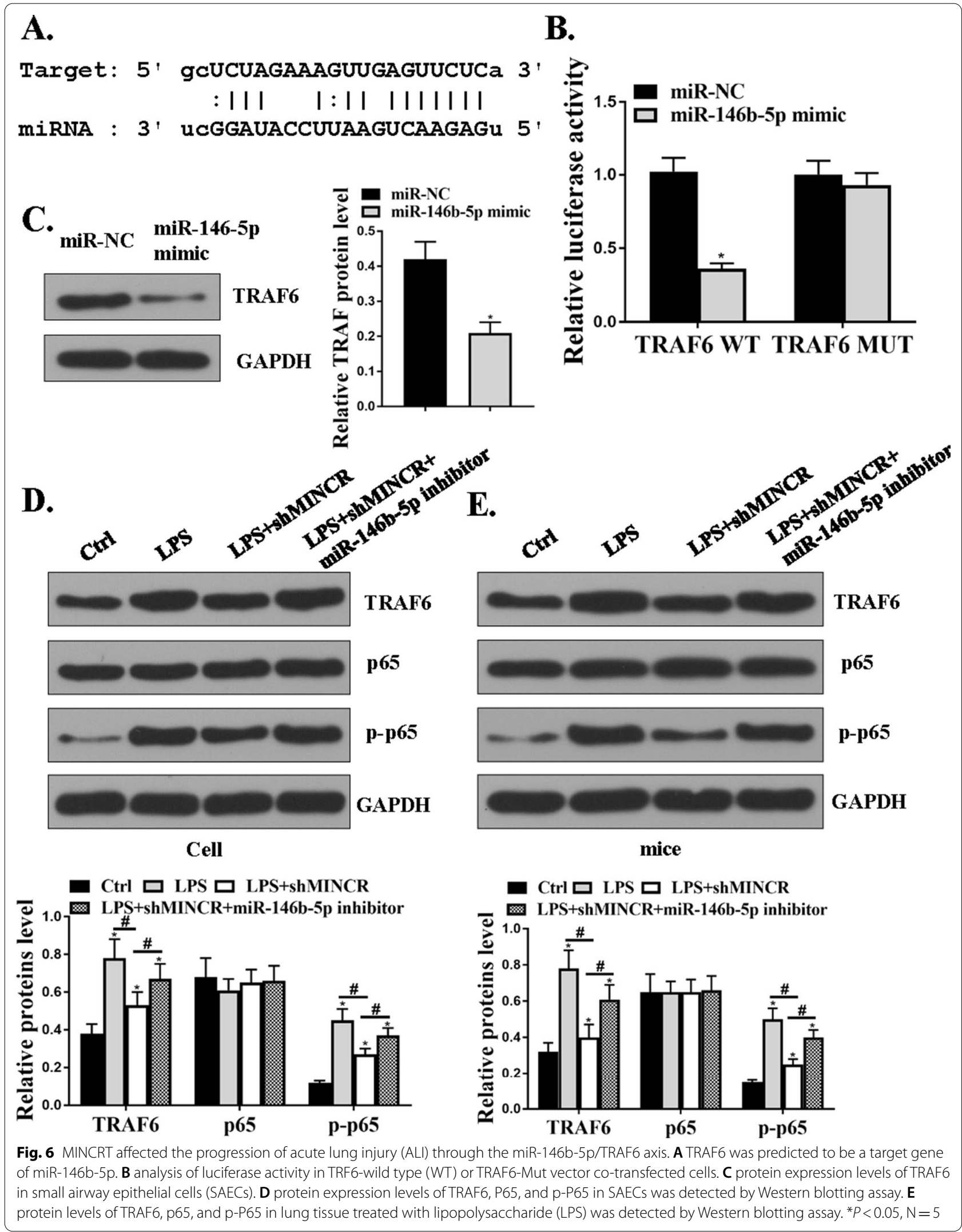


After LPS injection, there was a significant lung injury in the mice, indicating that ALI was successfully induced.

The biological functions of lncRNAs vary. They regulate the expression of specific genes in the process of recruitment, post-transcription modification, and epigenetics of transcription (Yuchen et al. 2020). Studies have shown that lncRNAs regulate gene expression at different levels, such as the transcriptional level of ALI, and participate in the development, infiltration, and transfer of ALI. For example, IncRNA MALAT1 is negatively regulated by BMI-111. Upregulation of BMI-111 can reduce the hypertonicity of endothelial cells by downregulating lncRNA MALAT1, reducing LPS-induced lung injury (Li et al. 2018b). In addition, an experimental model of pulmonary endothelial inflammation and barrier dysfunction was established by LPS stimulation of HPMECs. IncRNAs play a key role in LPS-induced pulmonary endothelial cell inflammation and barrier dysfunction ( $\mathrm{Li}$ et al. 2018c). MINCR is a recently discovered lncRNA. In this study, MINCR was found to be upregulated in the lung tissues of LPS-injured mice and SAECs. ShMINCR reduced LPS-induced lung tissue damage and the number of apoptotic cells in the lung tissue of mice. Sh-MINCR significantly reduced the total cell number, neutrophil number, MPO activity, F4/80 positive cell number, and the expression levels of inflammatory factors (TNF- $\alpha$ and IL-6) in LPS-induced BALF, and increased the expression levels of IL-10. After sh-MINCR treatment, the viability of SAECs increased and the apoptosis rate was reduced. It was speculated that MINCR plays a key role in the development of ALI inflammation caused by LPS, and that the knockout of PVT1 had a protective effect on LPS-induced ALI inflammation and cell viability.

Studies have shown that miRNAs are involved in regulating physiological processes, such as insulin secretion, tumor formation, bacterial infection, and viral infection (Li et al. 2019). It was reported that miRNAs play a role in lung diseases such as lung growth and development, pneumonia, lung cancer, and pulmonary fibrosis (Hou et al. 2018). For example, the expression levels of miR155 in the lung tissues of WT mice were significantly increased compared to those in miR-155 inhibitor mice. It can inhibit its expression and regulate the pulmonary inflammatory response by binding to the downstream target gene cytokine signal suppressor (Feng et al. 2016). As a member of the miRNA, miR-146b-5p plays different roles in different diseases (Xu et al. 2018). In this study, miR-146b-5p was identified as a target gene for MINCR. The expression levels of miR-146b-5p were significantly reduced in LPS-induced ALI lung tissues. Co-transfection of sh-MINCR with miR-146b-5p inhibitor increased the number of lung tissue damage and apoptosis.
Simultaneously, co-transfection of sh-MINCR with miR146b-5p inhibitor reversed the effects of sh-MINCR on the total cell number, neutrophil number, $\mathrm{F} 4 / 80$ positive cell number, the expression levels of inflammatory factors (TNF- $\alpha$, IL-6, and IL-10), and the activity and apoptosis of SAECs. These results indicate that MINCR regulates ALI injury through miR-146b-5p.

The tumor necrosis factor receptor related factor family (TRAF) is a conserved linker protein (Zhu et al. 2018). TRAF6 is closely related to ALI caused by sepsis, and overexpression of TRAF6 increases the expression levels of TNF- $\alpha$ and IL-6 (Wu et al. 2019b). Both p38 MAPK and NF- $\mathrm{KB}$ are involved in regulating the uncontrolled inflammatory response in AM (Tian et al. 2019). Activated P38 MAPK promotes the expression of AM and synthesizes and releases inflammatory transmitters and arachidonic acid metabolites, which are involved in the inflammatory process (Zhang et al. 2019). When $A M$ is stimulated by inflammation, NF- $\kappa B$ induces $A M$ to secrete a variety of inflammatory transmitters and aggravates lung injury (Wu et al. 2019a). Studies have shown that TRAF6 can activate the NF- $\mathrm{kB}$ signaling pathway (Ji et al. 2018). This study found that TRAF6 is a target gene of miR-146b-5p. Sh-MINCR reduced the expression levels of TRAF6 and p-P65 in LPS-induced SAECs and lung tissues of mice. Co-transfection of shMINCR with miR-146b-5p inhibitor reversed the effect of sh-MINCR on the expression of TRAF6 and p-P65. These data demonstrated that MINCR enhanced the expression levels of TRAF6 and p-P65 by regulating miR-146b-5p.

\section{Conclusion}

MINCR regulates ALI damage by upregulating the expression of TRAF6 and p-P65 through miR-146b-5p. Thus, it is a potential target for the treatment of ALI.

\begin{abstract}
Abbreviations
ALI: Acute lung injury; ARDS: Acute respiratory distress syndrome; BALF: Bronchoalveolar lavage fluid; ELISA: Enzyme-linked immunosorbent assay; FBS:

Fetal bovine serum; H\&E: Hematoxylin and eosin; IL: Interleukin; IncRNA: Long non-coding RNA; LPS: Lipopolysaccharide; WT: Wild type; MPO: Myeloperoxidase; PMNs: Polymorphonuclear leucocytes; qRT-PCR: Quantitative real-time polymerase chain reaction; SAECs: Small airway epithelial cells; TNF: Tumor necrosis factor; TNAF: Tumor necrosis factor receptor related factor family; TU: Transducing units; TUNEL: Terminal deoxynucleotidyl transferase biotin-dUTP nick end labeling.
\end{abstract}

\section{Acknowledgements}

This work was supported by Jinan Science and Technology Development Plan Project (Grant No. 201805067).

\section{Authors' contributions}

WG studied the concepts; designed the study; performed the literature research, clinical studies, experimental studies, data analysis; and wrote, edited, and reviewed the manuscript. YZ defined the intellectual content; studied 
the concepts; performed the literature research, clinical studies, experimental studies, data acquisition, statistical analysis, and data analysis; and prepared, wrote, and reviewed the manuscript. All authors read and approved the final manuscript.

\section{Funding}

This work was supported by the Jinan Science and Technology Development Plan Project (Grant No. 201805067).

\section{Availability of data and materials}

The data that support the findings of this study are available upon request from the corresponding author. The data are not publicly available because they contain information that can compromise the privacy of research participants.

\section{Declarations}

\section{Ethics approval and consent to participate}

All the experimental procedures performed in this study were approved by the Second Hospital of Shandong University Animal Ethics Committee. All animal experiments were performed in accordance with the "Guidelines for the Care and Use of Laboratory Animals" (revised in 1996, Publication No. 85-23) published by the National Institutes of Health. Nursing committee approval.

\section{Consent for publication}

Not applicable.

\section{Competing interests}

The authors declare that they have no competing interests.

\section{Author details}

'Department of Critical Care Medicine, The Second Hospital of Shandong University, Jinan 250033, Shandong, People's Republic of China. ${ }^{2}$ Department of Respiratory, The Second Hospital of Shandong University, No.247 Beiyuan Avenue, Jinan 250033, Shandong, People's Republic of China.

Received: 12 March 2021 Accepted: 31 August 2021

Published online: 03 October 2021

\section{References}

Bender TM, Stone LR, Amenta JS. Diagnostic power of lecithin/sphingomyelin ratio and fluorescence polarization assays for respiratory distress syndrome compared by relative operating characteristic curves. Clin Chem. 2020;40(4):541-5.

Cao C, Yin C, Shou S, Wang J, Chai Y. Ulinastatin protects against LPS-induced acute lung injury by attenuating TLR4/NF-KB pathway activation and reducing inflammatory mediators. Shock. 2018;50(5):595-605.

Chen Y, Wang D, Zhao Y, Huang B, Cao H, Qi D. p300 promotes differentiation of Th17 cells via positive regulation of the nuclear transcription factor RORgammat in acute respiratory distress syndrome. Immunol Lett. 2018;202:8-15.

Cheung OY, Graziano P, Smith ML. Acute lung injury. 2018.

Chiumello D, Marino A, Cammaroto A. The acute respiratory distress syndrome: diagnosis and management. 2019.

D'Alessio FR, Craig JM, Singer BD, Files DC, Mock JR, Garibaldi BT, et al. Enhanced resolution of experimental ARDS through IL-4-mediated lung macrophage reprogramming. Am J Physiol Lung Cell Mol Physiol. 2016;310(8):L733-46.

Ding $Y H$, Yun-duan S, Ya-xian W, Hui-qiong H, Tian-hong Y, Yu-dong H, et al. Isoalantolactone suppresses LPS-induced inflammation by inhibiting TRAF6 ubiquitination and alleviates acute lung injury. Acta Pharmacol Sin. 2019.

Emma R, Niku O, Terho LJCC. MicroRNAs in the Atherosclerotic Plaque. 2020;12:12.

Fava VM, Jeremy M, Aurélie C, Marianna O, Nguyen VT, Moraes MO, et al. A genome wide association study identifies a IncRna as risk factor for pathological inflammatory responses in leprosy. PLoS Genet. 2017;13(2):e1006637.
Feng T, Shao F, Wu Q, Zhang X, Xu D, Qian K, et al. miR-124 downregulation leads to breast cancer progression via LnCRNA-MALAT1 regulation and CDK4/E2F1 signal activation. Oncotarget. 2016;7(13):16205-16.

Feng Z, Qi S, Zhang Y, Qi Z, Yan L, Zhou J, et al. Ly6G + neutrophil-derived miR223 inhibits the NLRP3 inflammasome in mitochondrial DAMP-induced acute lung injury. Cell Death Dis. 2017;8(11):e3170.

Fu S, Thangavel S. Cardiac dysfunction in acute respiratory distress syndrome. Crit Care Nurs Q. 2019;42(4):448-58.

Gregoire M, Tadie JM, Uhel F, Gacouin A, Piau C, Bone N, et al. Frontline science: HMGB1 induces neutrophil dysfunction in experimental sepsis and in patients who survive septic shock. J Leukoc Biol. 2017;101(6):1281-7.

Gregoire M, Uhel F, Lesouhaitier M, Gacouin A, Guirriec M, Mourcin F, et al. Impaired efferocytosis and neutrophil extracellular trap clearance by macrophages in ARDS. Eur Respir J. 2018;52(2):1702590.

Hou T, Liao J, Zhang C, Sun C, Li X, Wang G. Elevated expression of miR-146, miR-139 and miR-340 involved in regulating Th1/Th2 balance with acute exposure of fine particulate matter in mice. Int Immunopharmacol. 2018;54:68-77.

Hu H, Hao L, Yan B, Li X, Zhu Y, Jin Y, et al. Long noncoding RNA MINCR regulates cellular proliferation, migration, and invasion in hepatocellular carcinoma. 2018.

lacona JR, Monteleone NJ, Lutz CS. miR-146a suppresses 5-lipoxygenase activating protein (FLAP) expression and Leukotriene B4 production in lung cancer cells. Oncotarget. 2018;9(42):26751.

Ji YF, Dan W, Liu YR, Ma XR, Zhang BA. MicroRNA-132 attenuates LPS-induced inflammatory injury by targeting TRAF6 in neuronal cell line HT-22: role of miR-132 in HT-22 cells. J Cell Biochem. 2018;119(7):5528-37.

Jian M, Kwan J, Bunting M, Ng R, Chan KH. Adiponectin suppresses amyloid- $\beta$ oligomer ( $A \beta O)$-induced inflammatory response of microglia via AdipoR1-AMPK-NF-kB signaling pathway. J Neuroinflamm. 2019;16(1):110.

Kanaya T, Sakakibara S, Jinnohara T, Hachisuka M, Ohno H. Development of intestinal $\mathrm{M}$ cells and follicle-associated epithelium is regulated by TRAF6-mediated NF-KB signaling. J Exp Med. 2018. https://doi.org/10. 1084/jem.20160659.

Li H, Shi H, Gao M, Ma N, Sun R. Long non-coding RNA CASC2 improved acute lung injury by regulating miR-144-3p/AQP1 axis to reduce lung epithelial cell apoptosis. Cell Biosci. 2018a;8(1):15.

Li H, Shi H, Ma N, Zi P, Liu Q, Sun R, et al. BML-111 alleviates acute lung injury through regulating the expression of InCRNA MALAT1. Arch Biochem Biophys. 2018b;649:15-21.

Li F, Sun J, Huang S, Su G, Pi G. LncRNA GAS5 overexpression reverses LPSinduced inflammatory injury and apoptosis through up-regulating KLF2 expression in ATDC5 chondrocytes. Cell Physiol Biochem. 2018c;45(3):1241-51.

Li H, Chen X, Wang Y, Yao D, Lin Y, et al. Exploration of the effect of blue light on microRNAs involved in the accumulation of functional metabolites of longan embryonic calli through RNA-sequencing. J Sci Food Agric. 2019;99:1533-47.

Liu J, Zhang Y, Lin D, Xiao R. Tanshinone IIA inhibits leukemia THP-1 cell growth by induction of apoptosis. Oncol Rep. 2009;21(4):1075-81.

Lyu Q, Jin L, Yang X, et al. LncRNA MINCR activates Wnt/ $\beta$-catenin signals to promote cell proliferation and migration in oral squamous cell carcinoma. 2019.

Ochroch EA, Wright GM, Riedel BJCJ. Anesthesia for open pulmonary resection: a systems approach. 2019.

Sanaa E, Seada LS. Quantitation of bcl-2 protein in bladder cancer tissue by enzyme immunoassay: comparison with Western blot and immunohistochemistry. Clin Chem. 2020;44(7):1423-9.

Sedgeman LR, Michell DL, Vickers KC. Integrative roles of microRNAs in lipid metabolism and dyslipidemia. Curr Opin Lipidol. 2019;30(3):1.

Sheng ZX, Yao H, Cai ZY. The role of miR-146b-5p in TLR4 pathway of glomerular mesangial cells with lupus nephritis. Eur Rev Med Pharmacol Sci. 2018;22:1737-43.

Tao H, Li N, Zhang Z, Mu H, Meng C, Xia H, et al. Erlotinib protects LPS-induced acute lung injury in mice by inhibiting EGFR/TLR4 signaling pathway. Shock. 2019;51:131-8.

Tian X, Xie G, Xiao H, Ding F, Bao W, et al. CXCR4 knockdown prevents inflammatory cytokine expression in macrophages by suppressing activation of MAPK and NF-KB signaling pathways. Cell Biosci. 2019;9:55.

Tong WJ. Basic and clinical research progress in acute lung injury/acute respiratory distress syndrome. Infect Int. 2018;7(2):38-43. 
Wang LK, Chen X-F, He D-D, Li Y, Fu J. Dissection of functional IncRNAs in Alzheimer's disease by construction and analysis of IncRNA-mRNA networks based on competitive endogenous RNAs. Biochem Biophys Res Commun. 2017;485(3):569-76

Wang C, Meng Y, Wang Y, Jiang Z, Xu M, Bo L, et al. Ouabain protects mice against lipopolysaccharide-induced acute lung injury. Med Sci Monit. 2018;24:4455.

Wu H, Wen F, Jiang M, Liu Q, Nie Y. LncRNA uc.48+ is involved in the diabetic immune and inflammatory responses mediated by $\mathrm{P} 2 \mathrm{X} 7$ receptor in RAW264.7 macrophages. Int J Mol Med. 2018. https://doi.org/10.3892/ ijmm.2018.3661.

Wu D, Liu J, Chen J, et al. MiR-449a suppresses tumor growth, migration and invasion in non-small cell lung cancer by targeting HMGB1-mediated NF-kB signaling way. 2019a.

Wu H, Yang TY, Li Y, Ye WL, Liu F, He XS, et al. TRAF6 promotes hepatocarcinogenesis by interacting with HDAC3 to enhance c-Myc gene expression and protein stability. Hepatology. 2019b. https://doi.org/10.1002/hep. 30801.

Xie G, Huang S, Luo Y, Ma L, Lin Z, Sun Y. LLCLPLDA: a novel model for predicting IncRNA-disease associations. Mol Genet Genomics. 2019;294(6):1477-86.

Xu T, Xie HQ, LiY, Xia Y, Rui S, Wang L, et al. Dioxin induces expression of hsa-miR-146b-5p in human neuroblastoma cells. J Environ Sci. 2018;63(1):260-7.
Yuchen L, Jingjing Z, Shulin Y, Zhen W, Xigan H, Yonghui S, et al. Extracellular vesicles long RNA sequencing reveals abundant mRNA, circRNA, and IncRNA in human blood as potential biomarkers for cancer diagnosis. Clin Chem. 2020;6(6):798-808.

Zhang M, Hu J, Li H, Zhang S. High TNF-a and/or p38MAPK expression predicts a favourable prognosis in patients with T1NOMO hepatocellular carcinoma: an immunohistochemical study. 2019.

Zhu T, Wang DX, Zhang W, Liao XQ, Guan X, Bo H, et al. Andrographolide protects against LPS-induced acute lung injury by inactivation of NF-kB. PLOS ONE. 2013;8(2):e56407.

Zhu Y, Wu G, Yan W, Zhan H, Sun P. miR-146b-5p regulates cell growth, invasion, and metabolism by targeting PDHB in colorectal cancer. Am J Cancer Res. 2017;7(5):1136.

Zhu S, Jin J, Gokhale S, Lu AM, Shan H, Feng J, et al. Genetic alterations of TRAF proteins in human cancers. Front Immunol. 2018;9:2111.

\section{Publisher's Note}

Springer Nature remains neutral with regard to jurisdictional claims in published maps and institutional affiliations.
Ready to submit your research? Choose BMC and benefit from:

- fast, convenient online submission

- thorough peer review by experienced researchers in your field

- rapid publication on acceptance

- support for research data, including large and complex data types

- gold Open Access which fosters wider collaboration and increased citations

- maximum visibility for your research: over 100M website views per year

At BMC, research is always in progress.

Learn more biomedcentral.com/submissions 